\title{
In vitro reconstructed human epithelia reveal contributions of Candida albicans EFG 1 and CPH1 to adhesion and invasion
}

Fraunhofer IGB, Nobelstr. 12, 70569 Stuttgart, Germany

\author{
C. Dieterich, M. Schandar, M. Noll,† F.-J. Johannes, H. Brunner, T. Graeve \\ and S. Rupp
}

Author for correspondence: S. Rupp. Tel: +49711970 4045. Fax: +497119704200.

e-mail: rup@igb.fhg.de

\begin{abstract}
The individual and synergistic contributions of two transcription factors, EFG1 and $C P H 1$, have been characterized with regard to adhesion to, and invasion of, human epithelia by Candida albicans. For this purpose two in vitro reconstructed tissue models were developed. A multi-layered model of human epidermis was used to simulate superficial infections of the skin, whereas a reconstructed human intestinal model was used to mimic the first steps of systemic infections. It was shown that $C$. albicans deleted for both transcription factors $C P H 1$ and EFG1, in contrast to the congenic clinical isolate Sc5314, was neither able to adhere to, nor to penetrate, either of the model systems. A strain deleted for EFG1 alone showed significant reduction in adhesion and was not able to penetrate through the stratum corneum. However, strains deleted for $\mathrm{CPH} 1$ showed phenotypes paralleling the phenotypes of the clinical isolate Sc5314. Using different types of multilayered human tissues reconstructed in vitro the individual contributions of Efg1p and Cph1p to two important virulence factors of C. albicans, namely adhesion and invasion, could be defined.
\end{abstract}

Keywords: fungal pathogen, yeast, dimorphism, test system

\section{INTRODUCTION}

Candida albicans is the most frequently isolated fungal pathogen in humans. It causes superficial infections of the skin, and mucous membranes, that may lead to lifethreatening, deep-seated systemic infections in immunocompromised patients. Since Candida naturally occurs in the gastrointestinal tract of humans it is considered an opportunistic pathogen, causing secondary infections (Odds, 1987). One main port of entry for systemic candidosis is through the epithelial layers of the gut. Invasion into blood vessels then leads to further dissemination of Candida throughout the body, resulting in a life-threatening systemic infection (Cole et al., 1996; Cutler, 1991).

Within the last few years our knowledge about the genes required for the virulence of Candida has increased dramatically, due to the ability to create deletions, or to introduce mutations, in the genome (Fonzi \& Irwin,

†Present address: IVB GmbH, Life Science Center, Schelztorstr. 54-56, 73728 Esslingen, Germany.

Abbreviation: FCS, fetal calf serum.
1993; Wilson et al., 1999). Mice are common animal models for determining the virulence of Candida strains for systemic or vaginal infections. However, infections of mice by Candida have not been reported to occur naturally (Ekenna \& Sherertz, 1987). To provide additional insights into the pathogenesis and treatment of fungal infections in vitro, systems mimicking infections of the human body as closely as possible can be important tools. Human cell systems reconstructed in vitro have already been used successfully to characterize Candida infections. For example, models of human cutaneous candidosis based on reconstructed human epidermis have been described (Korting et al., 1998; Schaller et al., 2000). Furthermore, endothelial, as well as epithelial, cell layers have been used to assess virulence mechanisms in C. albicans (Filler et al., 1995; Zink et al., 1996). Thus, in vitro systems have added significant new insights into the virulence mechanisms of Candida that can be gathered from animal models only with difficulty.

Two transcription factors have been described as being essential for virulence in C. albicans, EFG1 and CPH1. Candida deleted for both EFG1 and CPH1 has been 
shown to be avirulent in a mouse model of systemic infection (Lo et al., 1997) and in an immunosuppressed gnotobiotic piglet model (Andrutis et al., 2000). This strain, as well as a strain deleted for EFG1, also showed limited abilities to damage endothelial cells (Phan et al., 2000).

To further characterize the contributions of the individual transcription factors with regard to adhesion to and invasion of human tissue, we have developed two three-dimensional model systems. One model is composed of human enterocytes and fibroblasts, isolated from small intestine biopsies, that are embedded in a collagen matrix. The second model consists of human keratinocytes that differentiate on a collagen matrix containing dermal fibroblasts to an epidermal layer. Using both model systems we show that deletion of EFG1 in C. albicans leads to a significantly reduced ability to adhere to, or to invade, the epithelia, whereas deletions in CPH1 show much weaker phenotypes. In addition, we show that a strain defective for both efg1 and $c p h 1$ neither adheres to, nor invades, either of the human tissue models. Thus, even in the absence of detectable components of an immune system this strain is unable to damage host tissue.

\section{METHODS}

Candida strains. Strains used in this study were the clinical isolate Sc5314 (Fonzi \& Irwin, 1993), Can16 (cph1::hisG/ cph1: : hisG::URA3::hisG), Can33 (efg1::hisG/efg1: : hisG: :URA3:: hisG), Can34 (cph1:: hisG/cph1: : hisG, efg1: : his $G /$ efg 1 : : hisG::URA3:: hisG), Can37 (efg1::hisG/ efg1::hisG, EFG1::leu2-URA3) and Can38 (cph1::hisG/ cph1::hisG, efg1::hisG/efg1::hisG, EFG1-leu2-URA3) (Lo et al., 1997). Strains were cultured overnight in YPD (yeast extract/peptone/dextrose) medium containing 2\% glucose (Difco) from glycerol stock cultures, or plated onto YPD agar plates ( $2 \%$; Bacto Agar, Difco) for $48 \mathrm{~h}$ at $30{ }^{\circ} \mathrm{C}$. Growth during the invasion or adhesion assays was performed in the absence of antibiotics unless otherwise mentioned.

Reconstructed human epidermis. The reconstructed human epidermis was composed of dermal fibroblasts embedded in a biomatrix of collagen I, isolated from rat tails and keratinocytes. The keratinocytes differentiate during the cultivation process into a multi-layered epidermis with stratum corneum (Fig. 1a). Collagen I was isolated directly from rat tails (University of Hohenheim) after removal of the skin. The tails were frozen in liquid nitrogen, broken, and the collagen bundles were squeezed out and cleaned after thawing of the tail pieces. The isolated collagen was incubated for $1 \mathrm{~h}$ in $70 \%$ ethanol, washed with water and dissolved in $0.1 \%$ acetic acid for $2 \mathrm{~d}$ at $4{ }^{\circ} \mathrm{C}$. After centrifugation, for $1 \mathrm{~h}$ at $10000 \mathrm{~g}$, the supernatant containing the collagen was lyophilized and could be stored at $4{ }^{\circ} \mathrm{C}$, or dissolved in $0 \cdot 1 \%$ acetic acid at $4 \mathrm{mg} \mathrm{ml}^{-1}$ (gel matrix). Both the human keratinocytes and the epidermal fibroblasts were generated from foreskin. The dermis was dissected, cut into small pieces and incubated overnight with $1 \mathrm{U}$ dispase (Life Technologies) in PBS (Life Technologies) at $4{ }^{\circ} \mathrm{C}$. To isolate the keratinocytes, the epidermal tissue was removed from the dermis and incubated for $30 \mathrm{~min}$ at $37^{\circ} \mathrm{C}$ with trypsin $(2.5 \%$; GIBCO). The reaction was stopped by the addition of keratinocyte growth medium (KGM) plus $5 \%$ fetal calf serum (FCS) (Clonetics). After resuspension in $\mathrm{KGM}+5 \% \mathrm{FCS}$, cells were allowed to adhere for $4 \mathrm{~h}$ to
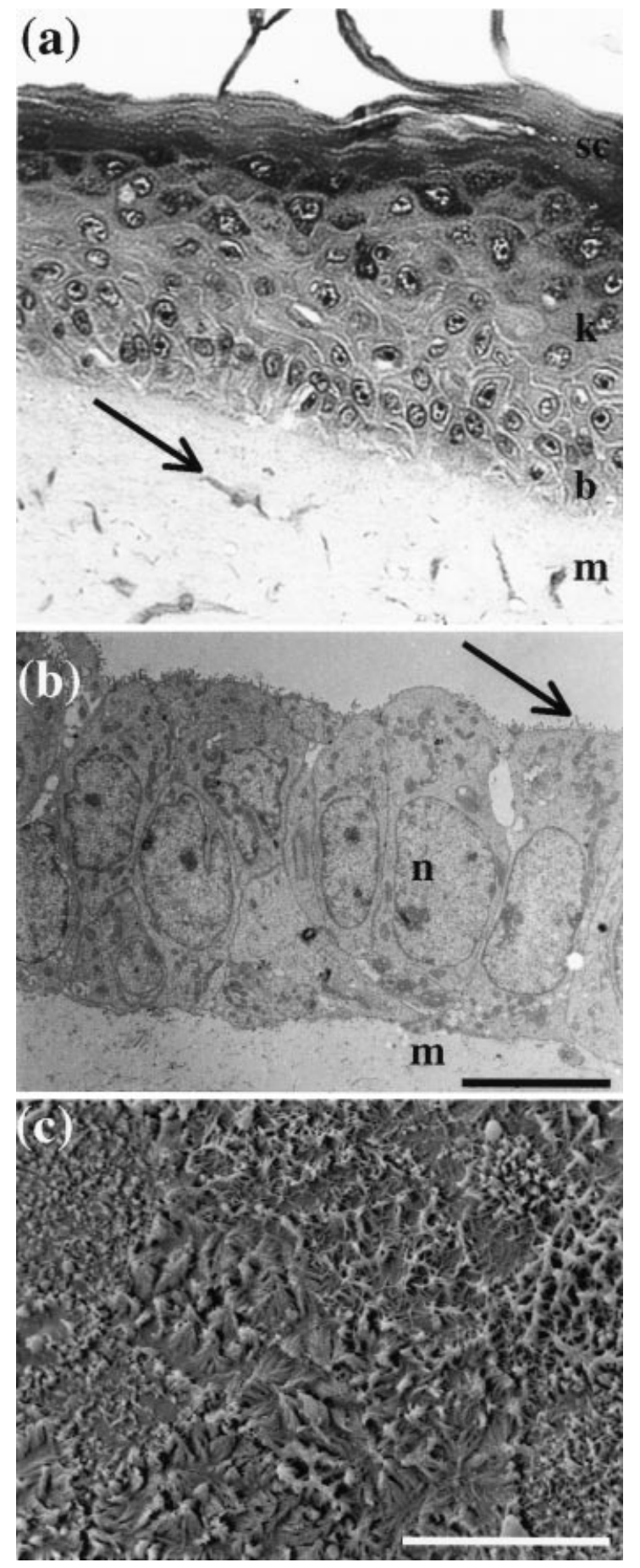

Fig. 1. In vitro reconstructed skin and intestinal equivalents. (a) Thin-section of the reconstructed epidermal model, consisting of the stratum corneum (sc), several layers of keratinocytes in different stages of differentiation (k), the basal lamina (b), and a collagen/fibroblast matrix $(\mathrm{m})$. The arrow indicates fibroblasts. (b) Transmission electron micrograph of the reconstructed intestinal model showing $\mathrm{CaCO} 2$ enterocytes forming a monolayer on top of a collagen/fibroblast matrix $(\mathrm{m})$. The nucleus (n) for each cell is clearly visible. Microvilli (indicated by the arrow) are visible on the apical membrane. Bar, $30 \mu \mathrm{m}$. (c) Scanning electron micrograph of a confluent cell layer of Caco2 cells grown on a collagen/fibroblast matrix differentiating microvilli. Bar, $20 \mu \mathrm{m}$. 
tissue-culture flasks coated with collagen I. The media was changed to remove tissue debris and thereafter every third day until the keratinocytes reached $80 \%$ confluency.

Epidermal fibroblasts were isolated by incubation of the human dermis for 45 min with $0.25 \%$ collagenase (GIBCO) in PBS $+2 \mathrm{mM} \mathrm{Ca}^{2+}$ and $2 \mathrm{mM} \mathrm{Mg}^{2+}$, at $37^{\circ} \mathrm{C}$. Cells were harvested and resuspended in M199+10\% FCS (GIBCO). Cells were allowed to adhere for $24 \mathrm{~h}$; the media was then changed to remove tissue debris. The medium was changed every third day until confluency was reached.

To generate the skin model, epidermal fibroblasts from primary culture were harvested and diluted with pre-cooled gel medium $\left[4{ }^{\circ} \mathrm{C} ; 2 \times\right.$ Dulbecco's Modified Eagle Medium (DMEM) containing $100 \mathrm{mM}$ HEPES (GIBCO)] to $5 \times 10^{5}$ cells $\mathrm{ml}^{-1}$. The cell suspension was carefully mixed with an equal volume of acidic collagen solution extracted from rat tails ( $4 \mathrm{mg} \mathrm{ml}^{-1}$ in $0 \cdot 1 \%$ acetic acid solution). Two hundred microlitres of this formulation was instantly poured into each cell-culture insert (12 $\mathrm{mm}$ diameter, polycarbonate membrane, $0.3 \mu \mathrm{m}$ pore size). The gels were allowed to solidify for $15 \mathrm{~min}$ at $37^{\circ} \mathrm{C}, 5 \% \mathrm{CO}_{2}$. After solidification, $50 \mu \mathrm{l}$ fibronectin $\left(5 \mu \mathrm{g} \mathrm{ml}^{-1}\right.$; Life Technologies) was spread onto the gels. Each insert was transferred to a cavity of a 24-well plate, provided with $1 \mathrm{ml}$ M199 medium (top and basolaterally) and equilibrated for $24 \mathrm{~h}$. On day2, the medium was replaced with $500 \mu \mathrm{l} \mathrm{KGM}+5 \%$ FCS basolaterally, and $500 \mu \mathrm{l} \mathrm{KBM}+5 \%$ FCS containing $1 \times 10^{5}$ keratinocytes gel $^{-1}$ was added to the inserts. The incubation medium was changed after the first day, thereafter every second day, using KGM $+2 \%$ FCS. After 4-6 d growth, under submerged conditions, the cell layer was exposed to air $\left(5 \% \mathrm{CO}_{2}\right)$ (airlift culture) to allow formation of a stratum corneum. For this purpose the insert was transferred to a 6-well plate, and airlift medium (KBM, without supplements, except $1.88 \mathrm{mM} \mathrm{Ca}^{2+}$ and $0.025 \mathrm{M}$ glucose) was added basolaterally only. The gels were cultivated for another 12-14 d, with daily change of medium, before use in the assay.

Epithelial cell-line culture. Three different intestinal cell lines, Caco2 (ATCC HTB-37), Lovo (ATCC CCL-229) and HT29 (ATCC HTB-38), were grown in $182 \mathrm{~cm}^{2}$ tissue-culture flasks (Greiner) and split 1:3, by standard methods, just before reaching confluency. All media were obtained from GIBCO. $\mathrm{Caco} 2$ cells were maintained in DMEM supplemented with $10 \%$ FCS, $2 \mathrm{mM}$ L-glutamine and $0 \cdot 1 \mathrm{mg}$ gentamicin $\mathrm{ml}^{-1}$. Lovo cells were grown in Ham's-F12 medium (Life Technologies) supplemented with $10 \%$ FCS and $0 \cdot 1 \mathrm{mg}$ gentamicin $\mathrm{ml}^{-1}$. HT29 cells were grown in McCoy's 5A medium (Life Technologies) supplemented with $10 \%$ fetal bovine serum, $2 \mathrm{mM}$ L-glutamin and $0 \cdot 1 \mathrm{mg}$ gentamicin $\mathrm{ml}^{-1}$. All cells were cultivated under standard conditions, $37^{\circ} \mathrm{C}, 5 \% \mathrm{CO}_{2}$. All antibiotics were omitted during co-cultivation with $\mathrm{C}$. albicans.

Primary culture of cells from human small intestine biopsies. All small intestine biopsies were obtained from clinical surgery at the Katharinen-Hospital, Stuttgart. Tissue samples were cut into small pieces of $1 \mathrm{~cm}^{2}$ and washed thoroughly in PBS. The mucosa was separated from the muscularis by mechanical means. Pieces of the small intestine muscularis were additionally treated for $1 \mathrm{~h}$ at $37^{\circ} \mathrm{C}$ with a $0.5 \%(\mathrm{w} / \mathrm{v})$ collagenase (crude extract; GIBCO) solution. Both components were washed in PBS again and placed in small tissueculture flasks. Cells were allowed to outgrow from tissue pieces under standard culture conditions for up to 1 week. Less than twofold proliferation of the isolated enterocytes was observed under various media conditions within 1 week. Human small intestine fibroblasts and myoblasts were grown with best results in FMK-2 medium (fibroblast medium kit, Sigma), with a doubling-time of about $24 \mathrm{~h}$, for up to $10 \mathrm{~d}$ (starting with $1 \times 10^{4}$ cells to yield $3-4 \times 10^{5}$ cells).

Reconstructed intestinal model. The three-dimensional intestinal model was generated over a $5 \mathrm{~d}$ period. At first, matrix cells, such as fibroblasts and myoblasts from primary culture, were harvested and diluted with pre-cooled $\left(4^{\circ} \mathrm{C}\right)$ gel medium $(2 \times$ DMEM containing $100 \mathrm{mM}$ HEPES $)$ to $5 \times 10^{5}$ cells $\mathrm{ml}^{-1}$. The cell suspension was carefully mixed with an equal volume of acidic collagen solution, extracted from rat tails (gel medium). Two hundred micorlitres of this formulation was instantly poured into each cell-culture insert $(12 \mathrm{~mm} \mathrm{di-}$ ameter); the gels were allowed to solidify for $15 \mathrm{~min}$ at $37^{\circ} \mathrm{C}$ in $5 \% \mathrm{CO}_{2}$. Each insert was transferred to a cavity of a 6 -well plate and provided basolaterally with $2 \mathrm{ml}$ DMEM medium. On day 3 the medium was replaced by fresh medium and $2 \times 10^{5} \mathrm{Caco} 2$ cells were placed on top of the gel. Gels were kept at $37^{\circ} \mathrm{C}, 5 \% \mathrm{CO}_{2}$, for at least $2 \mathrm{~d}$ to secure the formation of a confluent enterocytic layer (Fig. 1 b, c). The vitality of the epithelium was determined microscopically by using trypan blue. For infection assays, any remaining supernatant on the epithelium was removed to avoid outgrowth of Candida into the medium. A simplified intestinal model omitting the primary fibroblasts was also used.

Adhesion assay. The adhesion assay was set up in 24-well polystyrene plates (Greiner). The cavities were either untreated, or pretreated to establish a confluent monolayer of Caco 2 cells on the bottom of the well, using DMEM $+10 \%$ FCS medium.

To start the experiment, all culture medium was removed and $300 \mu \mathrm{l}$ testing medium (YPD or DMEM without antibiotics) was added to each well. The assay was incubated at $37^{\circ} \mathrm{C}, 5 \%$ $\mathrm{CO}_{2}$. After $1 \mathrm{~h} 10^{2}-10^{3} \mathrm{C}$. albicans cells from an overnight culture (grown in YPD at $30^{\circ} \mathrm{C}$ ) were added to each well. The exact inoculum was determined by c.f.u. counts on agar plates. After $0 \cdot 5,1,2$ and $3 \mathrm{~h}$ the assay plates were placed on a horizontal shaker for exactly 2 min at 200 r.p.m., and the contents of two cavities were spread onto agar plates. The proportion of non-adherent C. albicans cells was determined according to the c.f.u. counts. For YPD the c.f.u. counts had to be corrected for growth by counting the cells using a microscope, or by transferring all Candida to agar plates; with the exception of Can34, the cells in DMEM did not separate during the assay. The proportion of cells attached to the $\mathrm{Caco} 2$ cells was counted by removing the epithelial monolayer with $300 \mu \mathrm{l}$ PBS containing $0.02 \%(\mathrm{w} / \mathrm{v})$ EDTA. Each cavity was thoroughly rinsed and the cell suspension was spread onto agar plates to determine the c.f.u. count (Fig. 4c).

Cell numbers were normalized to the inoculum $(100 \%$ of cells) and corrected for growth. The c.f.u. count of the culture supernatant represented the proportion of non-adherent cells. Each value was based on at least three separate experiments conducted in duplicate.

Invasion assay. The epidermal and intestinal models described were used to test the ability of $C$. albicans strains to invade human tissue. For this purpose, the infection of the model was conducted by embedding yeast cells in DMEM medium solidified with $1 \%$ agar (final concentration of $10^{3}$ cells $\mathrm{ml}^{-1}$ ). Small agar slices were cut out with the end of a sterile pipette. The slices were carefully positioned on top of the epithelium and the cells were cocultivated under airlift conditions. The progress of infection was monitored by fixation of the tissue with acustain (Sigma) at time points of $0,18,24,48$ and $72 \mathrm{~h}$ post-infection. Paraffin sections $5 \mu \mathrm{m}$ thick were obtained with a Leitz microtome. To distinguish between all components of 

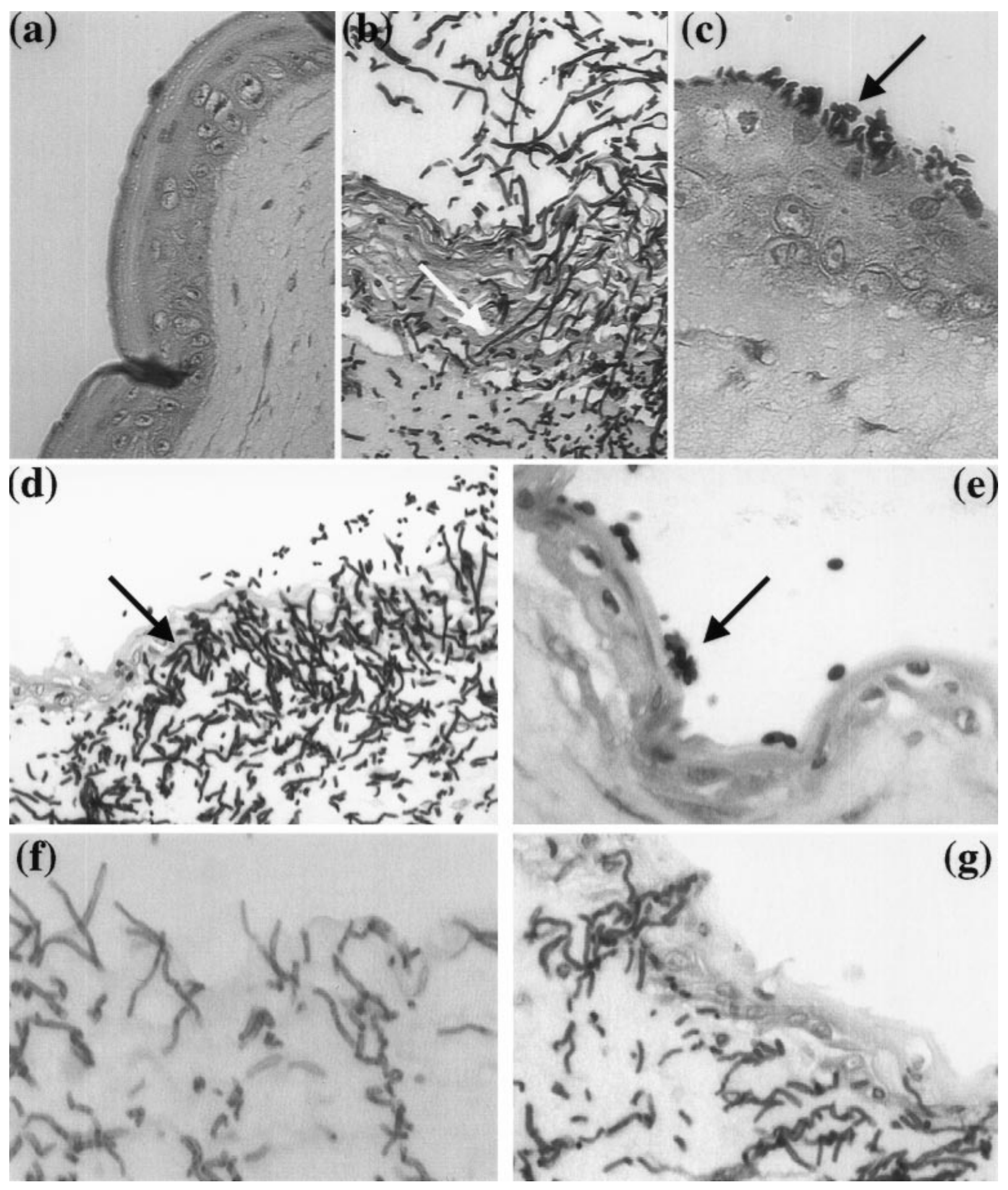

Fig. 2. Infection of a reconstructed skin equivalent with Sc5314, Can34 ( $\Delta$ cph1 $\Delta$ efg 1$)$, Can $16(\Delta c p h 1),(\Delta$ efg 1$)$, Can37 $(\Delta e f g 1, E F G 1)$ or Can38 $(\Delta c p h 1 \Delta e f g 1, E F G 1)$ for $48 \mathrm{~h}$ at $37^{\circ} \mathrm{C}$. The undisturbed reconstructed skin is shown in (a) (magnification $\times 200$ ). (b) Sc5314 penetrates the protective layer of keratinocytes and invades through the epithelial cell layers into the matrix, leading to severe damage of the model system. The arrow indicates hyphae (thread-like structures) (magnification $\times 200$ ). (c) Can34 is unable to penetrate or to damage the tissue, but superficially resides on top of the keratinocytic cell layer. Furthermore, Can34 does not form hyphae (see arrow) (magnification $\times 400)$. (d) Can16 ( $\Delta c p h 1)$ shows a similar phenotype to Sc5314. It forms hyphae that penetrate the epidermal layer and invade the collagen/fibroblast matrix (magnification $\times 200$ ). (e) Can33 ( $\Delta$ efg1), similar to Can34, is neither able to form hyphae nor to invade through the stratum corneum of the epidermal equivalent (magnification $\times 400)$. (f) Can37 ( $\Delta$ efg1, EFG1), containing a reintroduced copy of EFG1, shows invasion comparable to Sc5314 or Can16 ( $\Delta c p h 1)$ (magnification $\times 200$ ). (g) Can38 ( $\triangle c p h 1 \Delta$ efg1, EFG1), containing a reintroduced copy of EFG1, also invades in a manner similar to Can16 $(\Delta c p h 1)$ or Sc5314 (magnification $\times 200)$. 
the model system, the sections were stained sequentially with the PAS method of McManaus (1989) and the method of Papanicolaou (1989).

\section{RESULTS}

\section{Human epithelial test systems}

We used two in vitro reconstructed human epithelial systems to characterize the adhesive and invasive properties of C. albicans, representing a dermal and an intestinal equivalent. The dermal equivalent was composed of two cell types usually derived from circumcisions: dermal fibroblasts embedded in a collagen matrix, and epidermal keratinocytes that differentiated into a multi-layered epidermis including a stratum corneum (Fig. 1a). The embedded fibroblasts functioned in conditioning the medium and in facilitating differentiation of the keratinocytes in the reconstructed tissue. Similar to the dermal model, we also designed an intestinal equivalent. The model of the human gut consisted of cells derived from clinical biopsies of the small intestine. It was composed of a layer of collagen, embedding fibroblasts derived from the muscularis mucosae, and enterocytes. Cultivation of isolated enterocytes, however, did not generate sufficient amounts of cells for the assays (Grossmann et al., 1998). To establish a system that could be used for routine adhesion, invasion and drug-evaluation studies, we tested several enterocytic cell lines for growth rate, adhesion and confluency on the collagen/fibroblast matrix. Of the three cell lines tested (HT29, Lovo and $\mathrm{Caco} 2$ ) $\mathrm{Caco} 2$ showed the best performance with regard to adhesion to the collagen/fibroblast matrix, for differentiation to a compact cell layer and for generation of enough tissue for the assays. Electron microscopy studies showed a single cell layer that was ordered regularly and that differentiated microvilli on the apical membrane (Fig. 1b, c). No gaps between the cells were observed within the confluent cell layer (Fig. 1b, c).

\section{Avirulent C. albicans cannot penetrate the reconstructed tissues}

Two major virulence factors have been reported for $C$. albicans: adhesion and invasion. Two transcription factors, Cph1p and Efg1p, have been described to be essential for the virulence of C. albicans in a mouse model of systemic infection (Lo et al., 1997). To evaluate the individual contributions of the transcription factors Efg1p and Cph1p to invasion, we tested the ability of Candida strains deleted for one, or both, of the transcription factors to penetrate the two epithelial model tissues. For this purpose we exposed the reconstructed tissues described above to a defined number of cells $\left(10^{2}-10^{3}\right)$ from either Can34 ( $\left.\Delta c p h 1 \Delta e f g 1\right)$, Can33 $(\Delta e f g 1), \operatorname{Can} 16(\Delta c p h 1)$ or Sc5314 (clinical isolate). As a control, strains reverted for EFG1 [Can37 ( $\Delta e f g 1, E F G 1)$ and Can38 ( $\Delta c p h 1 \Delta e f g 1, E F G 1)]$ were included in this set of experiments. The Candida strains were embedded in soft agar and placed as a defined slice $(4 \mathrm{~mm}$ in diameter) on top of the reconstructed tissue, to set a precise infection. In the human dermal model we found strong invasion by the clinical isolate Sc5314 and by Can16 ( $\Delta c p h 1)$ after $48 \mathrm{~h}$ of infection (Fig. 2b, d). Invasion through the stratum corneum was already visible after $24 \mathrm{~h}$ for both of these strains (not shown). In

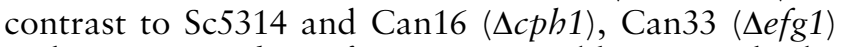
and Can34 $(\Delta c p h 1 \Delta e f g 1)$ were not able to invade the tissue of the reconstructed skin model, even after $48 \mathrm{~h}$ (Fig. 2c, e). Even prolonged periods of incubation, up to $72 \mathrm{~h}$, did not result in detectable invasion of the dermal model. However, reintegration of EFG1, as in Can37 $(\Delta e f g 1, E F G 1)$ and Can38 ( $\Delta c p h 1 \Delta e f g 1, E F G 1)$, resulted in induction of hyphae and invasion of the tissue as observed for Sc5314 and Can16 ( $\Delta c p h 1$ ) (Fig. 2f, g). This result reflected the avirulence of Can34 that was shown in a mouse model of systemic infection (Lo et al., 1997). Furthermore, it revealed that deletion of EFG1 alone was sufficient to block invasion through the stratum

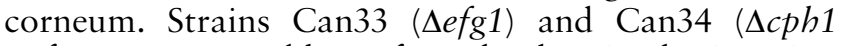
$\Delta e f g 1)$ were not able to form hyphae in the invasion assay (Fig. 2c, e). Deletion of CPH1 alone had no significant effect on the ability to invade the dermal model tissue when compared to Sc5314 (Fig. 2b, d).

To see if Can34 $(\Delta c p h 1 \Delta e f g 1)$ or Can33 ( $\Delta e f g 1)$ could penetrate through tissue not protected by a stratum corneum we tested if these strains would invade the reconstructed human intestinal model. The intestinal model did not contain a protective layer of cells at the apical surface and thus might be more susceptible to the pathogen. In contrast, it differentiated microvilli that increased the surface area available for Candida to adhere to. Again a defined number of cells $\left(10^{2}-10^{3}\right)$ of either Can34 ( $\Delta c p h 1 \Delta e f g 1)$, Can33 ( $\Delta e f g 1)$, Can16 $(\Delta c p h 1)$, or Sc5314, as well as Can37 ( $\Delta e f g 1, E F G 1)$ and Can38 ( $\Delta c p h 1 \Delta e f g 1, E F G 1)$, were fixed in agar and placed on top of the reconstructed intestinal tissue. After infection, Sc5314 and Can16 ( $\Delta c p h 1)$, as well as Can37 $(\Delta e f g 1, E F G 1)$ and Can38 ( $\Delta c p h 1 \Delta e f g 1, E F G 1)$, rapidly formed hyphae and penetrated the epidermal layer into the collagen matrix after $18 \mathrm{~h}$ (Fig. 3a, c, e, f, respectively). After $72 \mathrm{~h}, \mathrm{Sc5} 314$ and Can16 ( $\Delta c p h 1)$ had penetrated the entire depth of the tissue system and led to its disintegration (not shown). In contrast to the infection studies with the skin model, Can33 ( $\Delta e f g 1)$ was able to penetrate through the epithelial cell layer of the gut model by $18 \mathrm{~h}$ of infection (Fig. $3 \mathrm{~d}$ ); however, this strain did not invade significantly into the collagen matrix, even after $72 \mathrm{~h}$ of incubation (not shown). Thus, the infection by Can33 ( $\Delta e f g 1)$ was restricted mainly to the surface of the intestinal model. Can33 ( $\Delta e f g 1)$ was not able to form hyphae in this assay; however, pseudohyphal formation could be observed (Fig. 3d, indicated by an arrow). In contrast to the other strains tested, Can34 ( $\Delta c p h 1 \Delta e f g 1)$ was not able to penetrate through the epithelial cell layers (Fig. 3b), even after $72 \mathrm{~h}$ of incubation at $37^{\circ} \mathrm{C}$. Prolonged incubation of the strains led to exhaustion of the medium due to overgrowth of the Candida. Can34 ( $\Delta c p h 1 \Delta e f g 1)$ also was not able to induce hyphae. In contrast to Can34 ( $\Delta c p h 1$ 

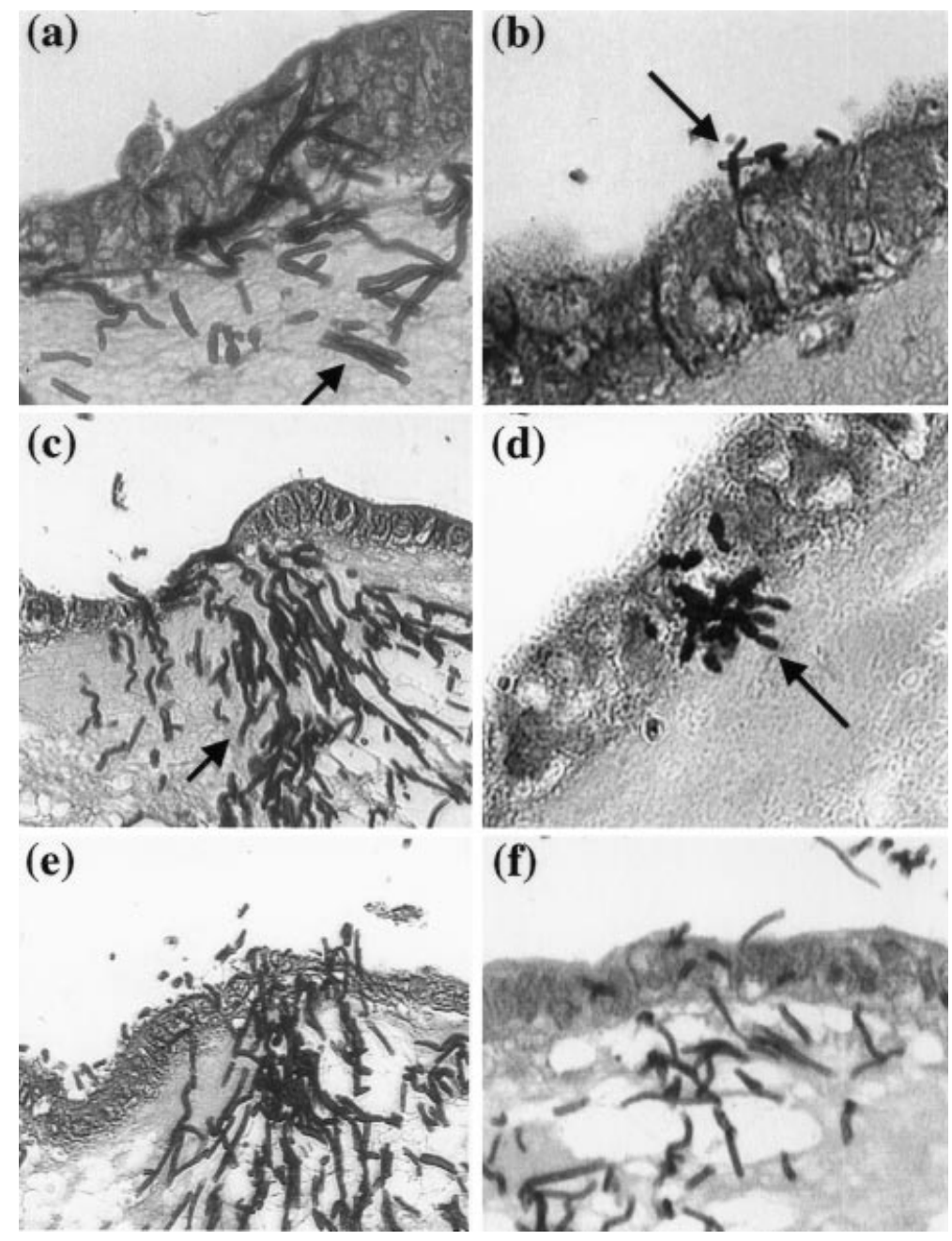

Fig. 3. Infection of the intestinal equivalent with Sc5314, Can34 ( $\Delta c p h 1 \Delta$ efg1), Can16 ( $\Delta c p h 1)$ Can33 ( $\Delta$ efg1), Can37 $\left(\Delta\right.$ efg1, EFG1) or Can38 ( $\Delta c p h 1 \Delta$ efg1, EFG1) for $18 \mathrm{~h}$ at $37^{\circ} \mathrm{C}$. (a) Rapid invasion of Sc5314 through the enterocytic cell layer into the collagen/fibroblast matrix (magnification $\times 400$ ). The individual hyphae can be seen growing through the entrocytes into the gel matrix (indicated by the arrow). (b) Can34 is not able to penetrate the model system. Only a few, non-hyphal, Candida cells (arrow) are visible on the enterocytic cell layer (magnification $\times 400)$. (c) Can16 ( $\Delta c p h 1)$ shows a phenotype similar to Sc5314. It forms hyphae that penetrate through the enterocytic cell layer and invade into the gel matrix (arrow) (magnification $\times 200)$. (d) Can33 $(\Delta e f g 1)$, in contrast to Can34 ( $\Delta c p h 1 \Delta$ efg1), is able to penetrate through the enterocytic cell layer, forming pseudohyphae (arrow) (magnification $\times 400$ ). Invasion into the gel matrix, however, is significantly reduced if compared to Can16 ( $\Delta c p h 1)$ (c) or Sc5314 (a). (e) Can37 ( $\Delta$ efg1, EFG1), containing a reintroduced copy of EFG1, shows invasion comparable to Sc5314 or Can16 ( $\Delta c p h 1)$ (magnification $\times 200)$. (f) Can38 ( $\Delta c p h 1 \Delta$ efg1, $E F G 1$ ), containing a reintroduced copy of EFG1, also invades in a similar way to Can16 ( $\Delta c p h 1)$ or Sc5314 (magnification $\times 200)$.

$\Delta e f g 1)$ and Can33 ( $\Delta e f g 1)$, Sc5314 and Can16 ( $\Delta c p h 1)$ readily formed hyphae that were able to penetrate through both the epidermal and enterocytic cell layers, into the gel matrix (Figs 2b, d and 3a, c). In histological thin-sections, Can34 could rarely be observed directly attached to the enterocytic or keratinocytic cell layer. Most likely this was due to reduced adhesion of Can34 $(\Delta c p h 1 \Delta e f g 1)$, which might have been removed during the extensive washing procedures in the course of the histological processing.

We also tested if invasion into agar, or collagen gel, lacking enterocytes could be observed. For this purpose we used a plate-wash assay as described by Roberts \&
Fink (1994). The strains described were grown on top of a collagen gel, or on DMEM agar plates (10\% serum). After $3 \mathrm{~d}$, the cell patches formed were washed away with water to visualize invasion by the different strains. Strains Sc5314, Can16, Can37 and Can38 invaded the agar, forming hyphae, whereas Can34 ( $\Delta c p h 1 \Delta e f g 1)$ was completely washed away. Can33 showed only a few pseudohyphal chains penetrating into the collagen, or agar, after $3 \mathrm{~d}$ at $37^{\circ} \mathrm{C}$, as observed for the intestinal model (not shown).

These results showed that even in the absence of any detectable component of the immune system Can34 $(\Delta c p h 1 \quad \Delta e f g 1)$ did not penetrate a single layer of 

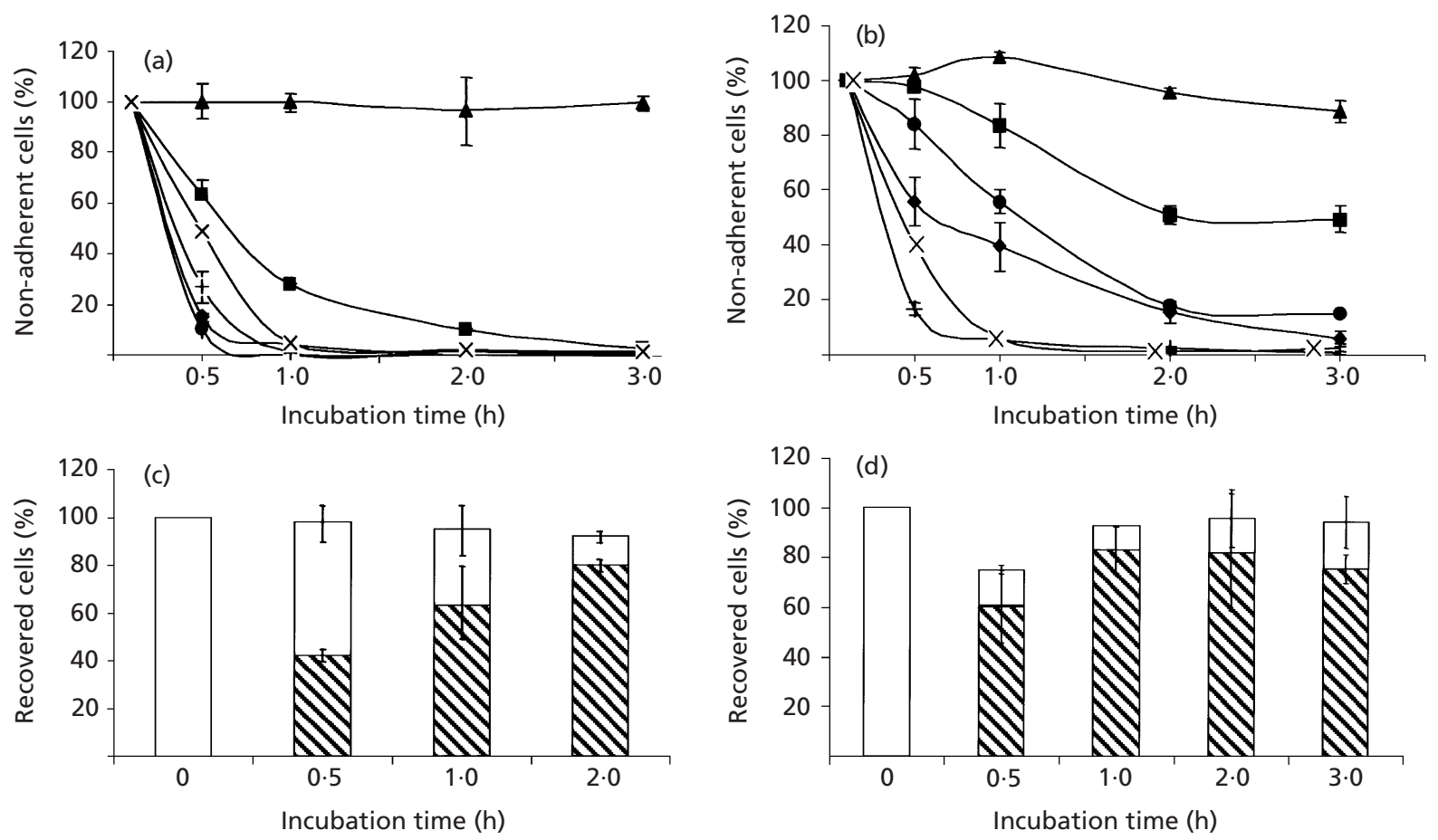

Fig. 4. Adhesion of Sc5314, Can16 ( $\Delta c p h 1)$, Can33 ( $\Delta$ efg1), Can34 ( $\Delta c p h 1 \Delta$ efg1), Can37 ( $\Delta$ efg 1, EFG1) and Can38 ( $\Delta c p h 1$ $\Delta$ efg1, EFG1) on polystyrene surfaces (a) or enterocytes (b). All the experiments were conducted, at least, as three

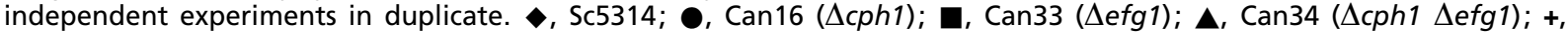
Can37 ( $\Delta$ efg1, EFG1); $\times$, Can38 ( $\Delta c p h 1 \Delta$ efg1, EFG1). Error bars indicate the SE for each set of experiments. (a) All strains incubated with DMEM $+10 \%$ FCS (at $37^{\circ} \mathrm{C}$ ) adhered to the polystyrene surface, with the exception of Can34, which did not adhere significantly, even after $3 \mathrm{~h}$ incubation. (b) Adhesion to enterocytes (Caco2) cultivated in DMEM $+10 \%$ FCS (at $37^{\circ} \mathrm{C}$ ). Sc5314, Can16, Can37 and Can38 strongly adhere to the epithelia, resulting in $<80 \%$ adhesive cells after $3 \mathrm{~h}$. Can33 shows significantly reduced adhesion to the Caco2 cells, leading to only $\sim 50 \%$ adhesion to the enterocytes after $3 \mathrm{~h}$ incubation. Can34 is not able to adhere significantly. (c) Overall recovery of Candida applied to Caco2 cells in the adhesion assays in DMEM + 10\% FCS. All of the Sc5314 cells applied in the assay can be accounted for, with a mean error over all time points of $10 \%$ (maximum error $23 \%$, minimum error $2 \%$ ). The white part of the bar indicates non-adherent cells recovered from the supernatant; the hatched part of the bar indicates adherent cells recovered after thoroughly washing the cells out of the plate. (d) Sc5314 shows adhesion to Caco2 cells after a $4 \mathrm{~h}$ pretreatment with 2 ug fluconazole $\mathrm{ml}^{-1}$ in YPD. After $30 \mathrm{~min}, 85 \%$ of the cells recovered adhered to the Caco2 cell layer in DMEM $+10 \%$ FCS, whereas only $15 \%$ could be recovered from the supernatant. The white part of the bar indicates non-adherent cells recovered from the supernatant; the hatched part of the bar indicates adherent cells recovered after thoroughly washing the cells out of the plate.

enterocytes, whereas a strain deleted for EFG1 still retained this ability. However, invasion into more rigid material, such as the collagen matrix or the stratum corneum, was restricted. Deletion of CPH1 had no detectable impact on invasion of the enterocytic cell layer as compared to Sc5314. Although there was no detectable effect of Cph1p alone, we clearly saw a synergistic effect if both Efg1p and Cph1p were missing in the cell, which was consistent with the in vivo results (Lo et al., 1997).

\section{Avirulent C. albicans cannot adhere to tissues or plastic surfaces}

Adhesion to the substrate has been shown to be a second important virulence factor besides invasion, as cells that had a reduced ability to adhere to tissue also showed reduced virulence (Gale et al., 1998; Muller et al., 1999; Tsuchimori et al., 2000). We tested whether strains defective in one, or both, of the transcription factors, CPH1 and EFG1, were defective in their adhesion to polystyrene surfaces, or to the enterocytes used in the intestinal model. Sc5314 adhered rapidly to both surfaces. On polystyrene surfaces the yeast-form cells [when grown in YPD (not shown)] as well as the germ-tubes [when grown in DMEM containing serum (Fig. 4a)] adhered to a similar extent. Thus, adhesion of Sc5314 was largely independent of the medium used. Adhesion to Caco2 cells also proceeded rapidly (Fig. 4b). Fig. 4(c) shows that we could recover all cells from the assay. Since Sc5314 forms germ-tubes in DMEM medium we did not observe an increase in the c.f.u. counts during the assay. To determine the contribution of the transcription factors individually, we measured the adhesive properties of strains lacking either $\mathrm{CPH} 1$ or EFG1. Strains lacking CPH1 showed an adhesion pattern on both polystyrene and enterocytes that was very similar to that of the wild-type cells. However, a strain deleted for EFG1 showed a more severe phenotype. Adhesion to polystyrene surfaces was similar to 
that seen for strains deleted for CPH1 or for wild-type cells (Fig. 4a), whereas adhesion to enterocytes was reduced to a level of about $50 \%$ of the wild-type adhesion (Fig. 4b). Thus, Efg1p again played a more pronounced role than Cph1p with regard to adhesion to host tissue. For Can34 ( $\Delta c p h 1 \Delta e f g 1)$, again, we saw significant additive effects of both transcription factors with regard to adhesion; Can34 was unable to adhere either to polystyrene surfaces or to tissue (Fig. 4a, b), independent of the medium used for cell culture. Reintegration of EFG1 into Can33, or Can34, reverted these phenotypes (Fig. 4a, b). These results could indicate a change in cell-wall composition of Can34 already in the yeast form as compared to the corresponding clinical isolate, Sc5314. Can34 thus lacked not only the ability to form hyphae, but also the ability to adhere to both host tissue and polystyrene, and had therefore lost two important virulence factors.

Adhesion of the non-pathogenic yeast Saccharomyces cerevisiae to polystyrene, under low-glucose conditions depending on the presence of the cell-surface protein Flo11p, was shown recently (Reynolds \& Fink, 2001). In contrast to C. albicans, neither haploid nor diploid $S$. cerevisiae ( $\Sigma 1278$ b background) adhered significantly to enterocytes in the assay described above (data not shown).

To investigate the effect of commonly used antimycotica on adhesion, we tested if treatment of Sc5314 with subinhibitory concentrations of fluconazole had an effect on adhesion to enterocytes. Sc5314 treated with fluconazole in DMEM $+10 \%$ serum was not able to form hyphae; this phenotype has been observed previously (Ha \& White, 1999). These cells, however, adhered to the enterocytes at least as well as the untreated cells (Fig. 4d). Thus, fluconazole did not inhibit adhesion of $C$. albicans to enterocytes.

\section{DISCUSSION}

Two virulence factors, adhesion and invasion, have been found to be central for pathogenesis of bacteria and fungi. Efg1p and Cph1p have been characterized as two transcription factors essential for virulence in $C$. albicans (Lo et al., 1997). To gain further insight into their individual contributions to adhesion and invasion, we developed two in vitro reconstructed human model tissues: a dermal model and an intestinal model. Both systems were composed of differentiated layers of human cells, grown on a collagen/fibroblast matrix. The main difference between the two systems, with regard to invasion, was the existence of a stratum corneum in the dermal model (Fig. 1). The stratum corneum has been reported to act as a barrier that could block invasion (Korting et al., 1998). However, Sc5314, the clinical isolate used in this study, has been shown to penetrate through the stratum corneum (Schaller et al., 1999). The reconstructed human intestine completely lacks a barrier like the stratum corneum, thus representing a more accessible system to pathogens. The model tissues described here contain an additional connective-tissue-like component, consisting of a collagen matrix and fibroblasts from the respective organ. Similar human tissue equivalents have been described (Korting et al., 1998; Schaller et al., 1999); however, these systems lack a collagen/fibroblast matrix. The embedded fibroblasts lead to conditioning of the medium and function like feeder cells to promote differentiation of the keratinocytes. The growth rates and number of cell layers, as well as stratification of the keratinocytes, was much less reproducible if fibroblasts, as feeder cells, were omitted from the skin model [see also Maruguchi et al. (1994) and Sugihara et al. (2001)]. Thus, the feeder cells ensure the quality of the skin model. For the intestinal model, growing the $\mathrm{Caco} 2$ cells on the collagen matrix with or without fibroblasts did not result in differences in the quality of the tissue nor in the adhesion or invasion of the Candida strains. However, the collagen matrix enabled us to visualize the invasion processes in more detail (see Fig. 3) and facilitated the handling of the tissues for histology. Further improvement of both systems to mimic the in vivo situation more closely could be achieved in the future by addition of components of the immune system into the collagen/fibroblast matrix, such as Langerhans cells or cells that are a first-defence line against fungal pathogens, e.g. neutrophils or macrophages, to generate simple aspects of an immune system.

Three congenic C. albicans strains deleted for CPH1, EFG1, or both transcription factors, as well as the respective reintegrants for EFG1, were compared to the clinical isolate Sc5314 with regard to invasion of, and adhesion to, the model tissues described above. Our results showed that a strain deleted for CPH1 behaved similarly to the corresponding clinical isolate Sc5314, whereas a strain deleted for EFG1 was significantly impaired for both adhesion and invasion. Can16 $(\Delta c p h 1)$, as well as Sc5314, penetrated as hyphae into both the intestinal and dermal equivalents within 18$24 \mathrm{~h}$, whereas Can33 ( $\Delta e f g 1$ ) was not able induce hyphae or to invade into the stratum corneum of the dermal model, even after $72 \mathrm{~h}$. However, pseudohyphal invasion through the enterocytic cell layer of the intestinal model could be observed (Fig. 3d). Thus, Can33 ( $\Delta e f g 1$ ) showed only limited invasive abilities, which might reflect the reduced virulence of this strain in a mouse model of systemic infection (Lo et al., 1997). In addition to the reduced invasive potential of Can33 ( $\Delta e f g 1)$, its adhesion to enterocytes was reduced to $50 \%$ as compared to Sc5314. EFG1 has been reported to be the major regulator of $H W P 1$, a cell-wall protein that contributes about $50 \%$ to the overall adhesion to human buccal epithelial cells (Staab et al., 1999). This parallels the reduced adhesion of Can33 ( $\Delta e f g 1)$ to enterocytes. Lack of Hwp1p also led to reduced virulence in a mouse model of systemic candidiosis, indicating the importance of adhesion for virulence (Staab et al., 1999; Tsuchimori et al., 2000). Thus, the lack of Hwp1p could explain the observed phenotypes in part. However, $\Delta h w p 1$ strains still form hyphae (Staab et al., 1999), implying that additional pathways contribute to virulence that are 
defective in $\Delta e f g 1$ strains. The adhesion of Can33 ( $\Delta e f g 1)$ to polystyrene was only slightly reduced if compared to Sc5314. This might indicate that different cell-surface components are required for adhesion to plastic surfaces than to human tissue.

Deletion of both CPH1 and EFG1, as in Can34 ( $\Delta c p h 1$ $\Delta e f g 1)$, had strongly additive effects. Can34 ( $\Delta c p h 1$ $\Delta e f g 1)$ did not adhere significantly to the tissues or to the plastics tested, and was completely non-invasive in both epithelia. This is consistent with observations in other systems reporting that $\Delta c p h 1 \Delta e f g 1$ strains were observed to cause only limited damage to tissue (Andrutis et al., 2000; Phan et al., 2000). Although Cph1p does not seem to be required for invasion or adhesion in Can16 $(\Delta c p h 1)$, it contributes significantly to the remaining invasive and adhesive properties of Can33 ( $\Delta$ efg1). The complete lack of adhesion of Can34 ( $\Delta c p h 1 \Delta e f g 1)$ is also reflected in the limited presence of the mutant cells on the tissue after histological processing (Figs $2 \mathrm{c}$ and $3 \mathrm{~b})$. This newly discovered absence of adhesion to host tissue might be another reason, besides the inability to form hyphae, for the strongly reduced virulence of this strain. The phenotypes observed in strains with deletions in EFG1 could be reversed after reintegration of EFG1.

For endothelial cells it has been shown that endocytosis is one of the main mechanisms of C. albicans invasion (Filler et al., 1995). In fact, $\Delta e f g 1$ strains, and to a lesser extent $\Delta c p h 1 \Delta e f g 1$ strains, have been shown to be taken up by endocytosis into endothelial cells (Phan et al., 2000); a similar mechanism has been suggested for epithelial cells (Drago et al., 2000). Thus, $\Delta e f g 1$ strains could penetrate not only by mechanical/enzymic force, but also by transcytosis. Stratified keratinocytes, however, lost their ability for endocytosis. This might be another explanation why we observed penetration of $\Delta e f g 1$ strains through the intestinal model, but not through the skin model.

We observed that fluconazole did not repress adhesion of Sc5314 to epithelial cells (Fig. 4d). Since Sc5314 was no longer able to form hyphae in the presence of fluconazole (Ha \& White, 1999), adhesion, formation of hyphae and invasion into the host tissue are distinguishable processes. Thus it is important to monitor these processes individually.

Our studies reveal that the reconstructed epithelia presented here can be used to illustrate the infection process of human tissue and to differentiate between the virulence of the individual Candida strains used. Due to the three-dimensional nature of these tissue models each step required for infection, from adhesion to penetration of the tissue, could be monitored. These results not only reflect the results obtained with distinct model systems (Andrutis et al., 2000; Lo et al., 1997; Phan et al., 2000), but add additional information to the contributions of Efg1p or Cph1p to both adhesion and invasion. For Can34 ( $\Delta c p h 1 \Delta e f g 1)$ we could show that it was devoid of both detectable invasive and adhesive properties. This resulted in the inability to damage even unprotected enterocytes, devoid of detectable components of the immune system. These results suggest that blocking virulence mechanisms in pathogens might inhibit host infections, even in severely immunocompromised patients. This opens the possibility for a much bigger selection of targets for the development of antimycotica than targeting essential genes only.

\section{ACKNOWLEDGEMENTS}

This work was supported by grant no. 03121805 from the Bundesministerium für Bildung und Forschung. We thank Michael Schweikert for the transmission electron micrograph, Wolfgang Gumbert for histology, Michaela Weimer and Matthias Rottmann for support in tissue culture, Kai Sohn for critical reading of the manuscript and Gerald Fink for providing C. albicans strains.

\section{REFERENCES}

Andrutis, K. A., Riggle, P. J., Kumamoto, C. A. \& Tzipori, S. (2000). Intestinal lesions associated with disseminated candidiasis in an experimental animal model. J Clin Microbiol 38, 2317-2323.

Cole, G. T., Halawa, A. A. \& Anaissie, E. J. (1996). The role of the gastrointestinal tract in hematogenous candidiasis: from the laboratory to the bedside. Clin Infect Dis 22 Suppl 2, S73-88.

Cutler, J. E. (1991). Putative virulence factors of Candida albicans. Annu Rev Microbiol 45, 187-218.

Drago, L., Mombelli, B., De Vecchi, E., Bonaccorso, C., Fassina, M. C. \& Gismondo, M. R. (2000). Candida albicans cellular internalization: a new pathogenic factor? Int J Antimicrob Agents 16, 545-547.

Ekenna, O. \& Sherertz, R. J. (1987). Factors affecting colonization and dissemination of Candida albicans from the gastrointestinal tract of mice. Infect Immun 55, 1558-1563.

Filler, S. G., Swerdloff, J. N., Hobbs, C. \& Luckett, P. M. (1995). Penetration and damage of endothelial cells by Candida albicans. Infect Immun 63, 976-983.

Fonzi, W. A. \& Irwin, M. Y. (1993). Isogenic strain construction and gene mapping in Candida albicans. Genetics 134, 717-728.

Gale, C. A., Bendel, C. M., McClellan, M., Hauser, M., Becker, J. M., Berman, J. \& Hostetter, M. K. (1998). Linkage of adhesion, filamentous growth, and virulence in Candida albicans to a single gene, INT1. Science 279, 1355-1358.

Grossmann, J., Maxson, J. M., Whitacre, C. M., Orosz, D. E., Berger, N. A., Fiocchi, C. \& Levine, A. D. (1998). New isolation technique to study apoptosis in human intestinal epithelial cells. Am J Pathol 153, 53-62.

Ha, K. C. \& White, T. C. (1999). Effects of azole antifungal drugs on the transition from yeast cells to hyphae in susceptible and resistant isolates of the pathogenic yeast Candida albicans. Antimicrob Agents Chemother 43, 763-768.

Korting, H. C., Patzak, U., Schaller, M. \& Maibach, H. I. (1998). A model of human cutaneous candidosis based on reconstructed human epidermis for the light and electron microscopic study of pathogenesis and treatment. J Infect 36, 259-267.

Lo, H. J., Kohler, J. R., Di Domenico, B., Loebenberg, D., Cacciapuoti, A. \& Fink, G. R. (1997). Nonfilamentous C. albicans mutants are avirulent. Cell 90, 939-949.

McManaus, G. N. (1989). Darstellung von paraplasmatischen substanzen, PAS reaktion nach McManaus. In Romeis, Mikroskopische Technik, p. 394. Edited by P. Böck. München, Wien, Baltimore: Urban and Schwarzenberg. 
Maruguchi, T., Maruguchi, Y., Suzuki, S., Matsuda, K., Toda, K. \& Isshiki, N. (1994). A new skin equivalent: keratinocytes proliferated and differentiated on collagen sponge containing fibroblasts. Plast Reconstr Surg 93, 537-544; discussion, 545-546.

Muller, F. M., Morschhauser, J., Kohler, G., Oelschlaeger, T. A., Ziebuhr, W. \& Hacker, J. (1999). Adherence and invasion - two pathogenicity factors in bacterial and fungal pathogens. Mycoses 42, 39-42.

Odds, F. C. (1987). Candida infections: an overview. Crit Rev Microbiol 15, 1-5.

Papanicolaou, J. F. A. (1989). Färbetechniken der zytodiagnostik, färbung nach Papanicolaou. In Romeis, Mikroskopische Technik, p. 646. Edited by P. Böck. München, Wien, Baltimore: Urban and Schwarzenberg.

Phan, Q. T., Belanger, P. H. \& Filler, S. G. (2000). Role of hyphal formation in interactions of Candida albicans with endothelial cells. Infect Immun 68, 3485-3490.

Reynolds, T. B. \& Fink, G. R. (2001). Bakers' yeast, a model for fungal biofilm formation. Science 291, 878-881.

Roberts, R. L. \& Fink, G. R. (1994). Elements of a single MAP kinase cascade in Saccharomyces cerevisiae mediate two developmental programs in the same cell type: mating and invasive growth. Genes Dev 8, 2974-2985.

Schaller, M., Preidel, H., Januschke, E. \& Korting, H. C. (1999). Light and electron microscopic findings in a model of human cutaneous candidosis based on reconstructed human epidermis following the topical application of different econazole formulations. J Drug Target 6, 361-372.

Schaller, M., Schackert, C., Korting, H. C., Januschke, E. \& Hube, B. (2000). Invasion of Candida albicans correlates with expression of secreted aspartic proteinases during experimental infection of human epidermis. J Invest Dermatol 114, 712-717.

Staab, J. F., Bradway, S. D., Fidel, P. L. \& Sundstrom, P. (1999). Adhesive and mammalian transglutaminase substrate properties of Candida albicans Hwp1. Science 283, 1535-1538.

Sugihara, H., Toda, S., Yonemitsu, N. \& Watanabe, K. (2001). Effects of fat cells on keratinocytes and fibroblasts in a reconstructed rat skin model using collagen gel matrix culture. $\mathrm{Br} J$ Dermatol 144, 244-253.

Tsuchimori, N., Sharkey, L. L., Fonzi, W. A., French, S. W., Edwards, J. E., Jr \& Filler, S. G. (2000). Reduced virulence of HWP1-deficient mutants of Candida albicans and their interactions with host cells. Infect Immun 68, 1997-2002.

Wilson, R. B., Davis, D. \& Mitchell, A. P. (1999). Rapid hypothesis testing with Candida albicans through gene disruption with short homology regions. J Bacteriol 181, 1868-1874.

Zink, S., Nass, T., Rosen, P. \& Ernst, J. F. (1996). Migration of the fungal pathogen Candida albicans across endothelial monolayers. Infect Immun 64, 5085-5091.

Received 5 July 2001; revised 17 October 2001; accepted 18 October 2001. 